\title{
Software para el modelamiento, simulación y programación de aplicaciones robotizadas
}

\begin{abstract}
RESUMEN
Actualmente existe una gran variedad de software que permiten simular celdas robotizadas e interactuar con un proceso. En este artículo se detalla el procedimiento para simular y programar un sistema robotizado de clasificación de piezas empleando el software Cosimir Educational. En el proyecto se incluye una ventana de modelamiento, posiciones y programación. La programación se realizó empleando el lenguaje Mitsubishi Robot Language (MRL), el cual es sencillo pero con suficiente capacidad para realizar tareas con el robot. Este lenguaje tiene comandos de control de movimientos, contadores, temporizadores, subrutinas, bucles e incorpora señales de inputs y outputs.
\end{abstract}

Palabras clave: Software, modelamiento, simulación y programación, aplicaciones robóticas.

SOFTWARE FOR MODELING, SIMULATION AND PROGRAMMING ROBOTIC APPLICATIONS

\section{ABSTRACT}

Currently there is a wide range of software to simulate robotic cell and interact with a process. This article details the procedure to simulate and program a robotic system of classification of items using the software Cosimir Educational. The project includes a window model, positions and programming. Programming is performed using the language Mitsubishi Robot Language (MRL), which is simple but with enough capacity to perform tasks with the robot. This language has commands for movement control, counters, timers, subroutines, loops and incorporates input and output signals.

KEYWORDS: Software, modeling, simulation and programming, robotic applications.

\section{INTRODUCCIÓN}

No es raro oír sobre la implementación de robots en las diversas aplicaciones en la industria mundial. Existe una gran variedad de modelos y sistemas robotizados como también una gran cantidad de empresas que los proveen. Estas empresas tienen a su disposición software de modelamiento que les permite simular la manera en que una aplicación robotizada funcionará dentro de una planta de producción automatizada, para facilitar de esta manera la tarea de toma de decisiones y elegir o no la adecuación o creación de un sistema robotizado. Para tal efecto, el software de simulación permite mostrar de manera gráfica y simulada como quedará un sistema de producción, incluyendo obviamente dentro de líneas de producción brazos robóticos que ejecutarán operaciones de ensamble, soldadura, pintura, moldeado, transferencia de materiales, clasificación de piezas, etc. Es importante mencionar que en internet hay posibilidad de descargar, a modo de evaluación y de manera gratuita, software de simulación y modelamiento de robots.

\section{SOFTWARE DE SIMULACIÓN}

Una de las tantas aplicaciones que permiten este tipo de simulaciones es el software Cosimir Educational con el que se puede modelar, diseñar y simular sistemas de producción robotizados.

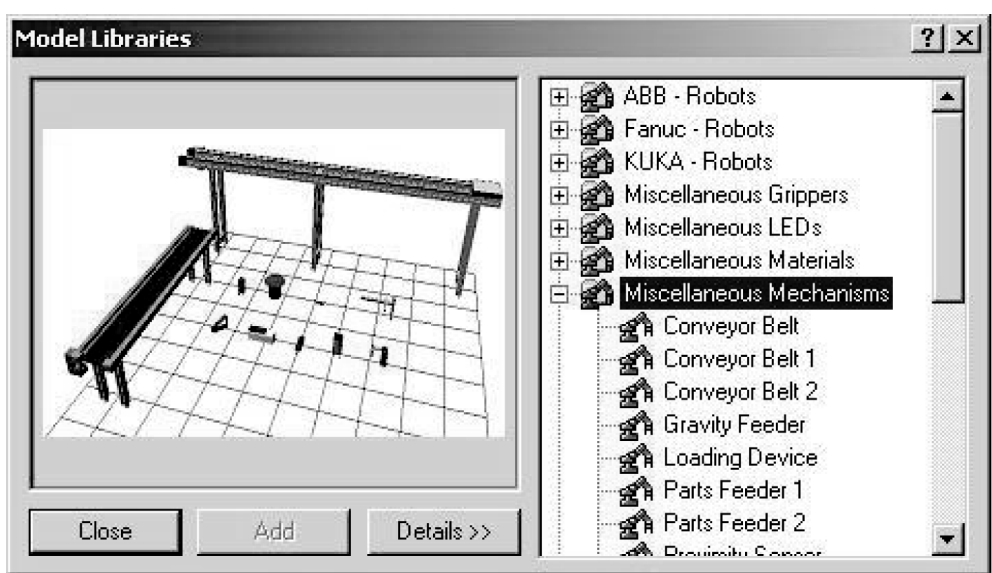

Fig. 1: Librería del Cosimir Educational.

* Email: avelasquez@urp.edu.pe; cim.urp@gmail.com Jefe del Laboratorio de Automatización - CIM (URP) 
Este software está disponible en la web para que toda persona interesada en el tema lo pueda evaluar por un determinado periodo de tiempo. Posee una amigable interfase de diseño y programación que permite un fácil manejo. Asimismo, cuenta con una librería de elementos que contiene modelos de robots, pinzas, herramientas, alimentadores neumáticos, sensores, PLC's, etc. Ver Fig. 1 (Librería del Cosimir Educational)

Al abrir el software aparecen varias ventanas, entre las principales están la ventana de modelamiento, de posiciones y de programación. Ver Fig. 2 (Vista del software Cosimir Educational).

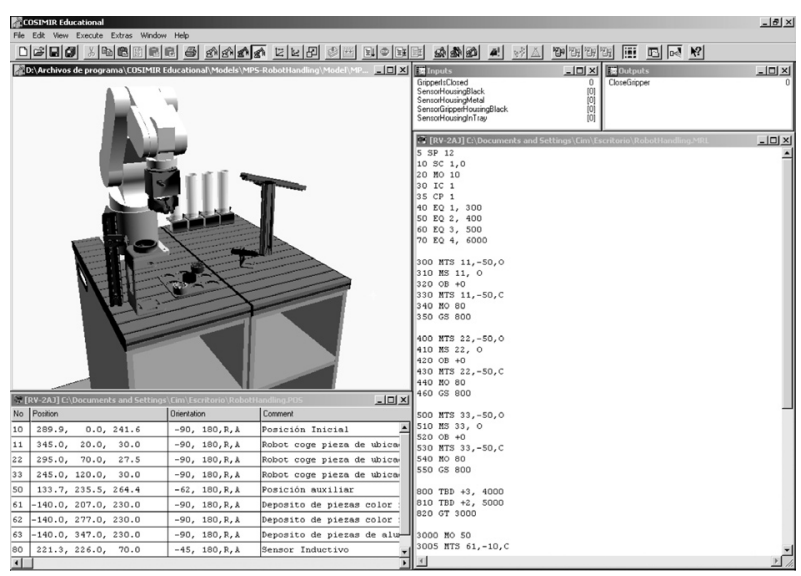

Fig. 2: Vista del software Cosimir Educational.

\section{VENTANA DE MODELAMIENTO}

En la ventana de modelamiento se puede incorporar cualquier objeto que se encuentre en la librería del software. Ver Fig. 3 (Ventana de modelamiento). Estos objetos poseen propiedades que pueden ser fácilmente modificadas algunas de estas propiedades son la dimensión del objeto, posición que va a tener el objeto dentro del entorno de trabajo y color del objeto. Este modelamiento se realiza en 3D. Ver Fig. 4 (Modelamiento de una fábrica).

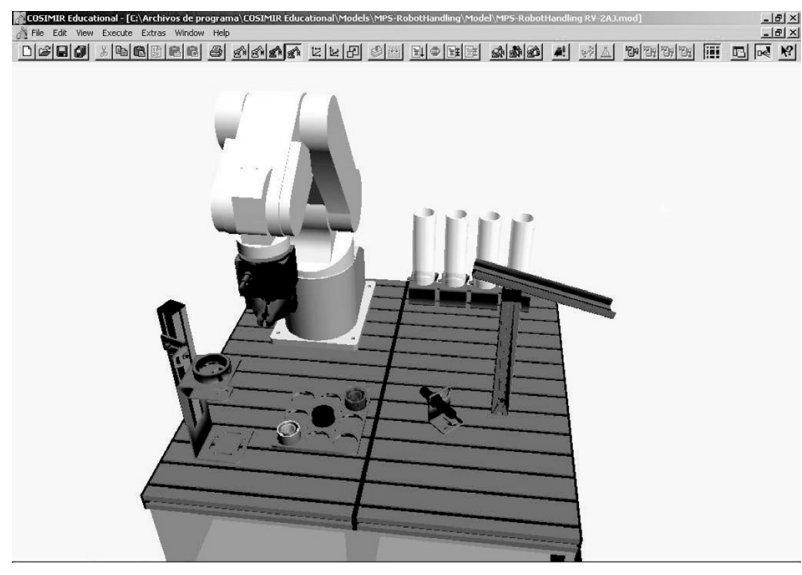

Fig. 3: Ventana de modelamiento.

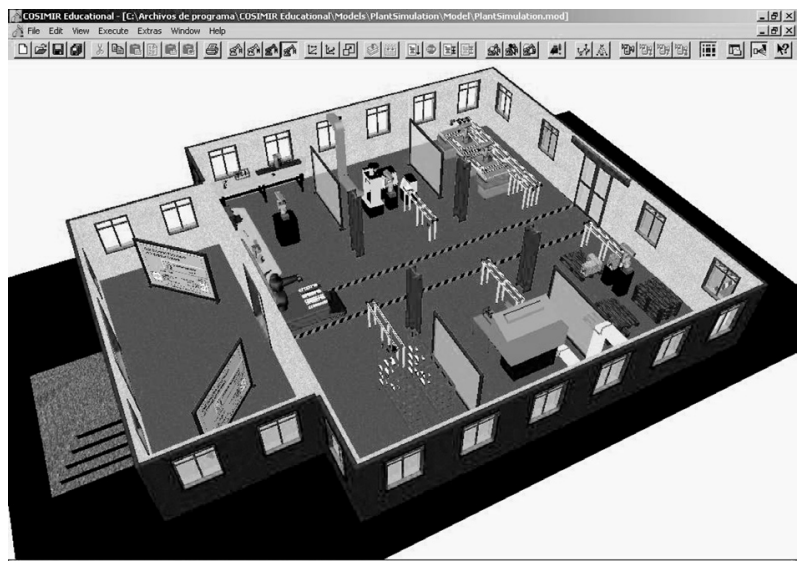

Fig. 4: Modelamiento de una fábrica.

\section{VENTANA DE POSICIONES}

La ventana de posiciones se emplea para grabar y almacenar la información de las coordenadas a través de las cuales el robot deberá moverse. Las coordenadas están dadas en valores numéricos y en $\mathrm{mm}$. Además cada posición tiene la información de la orientación que tiene la pinza u herramienta del robot así como también información de si el robot tiene las pinzas abiertas o cerradas. Ver Fig. 5 (Ventana de posiciones).

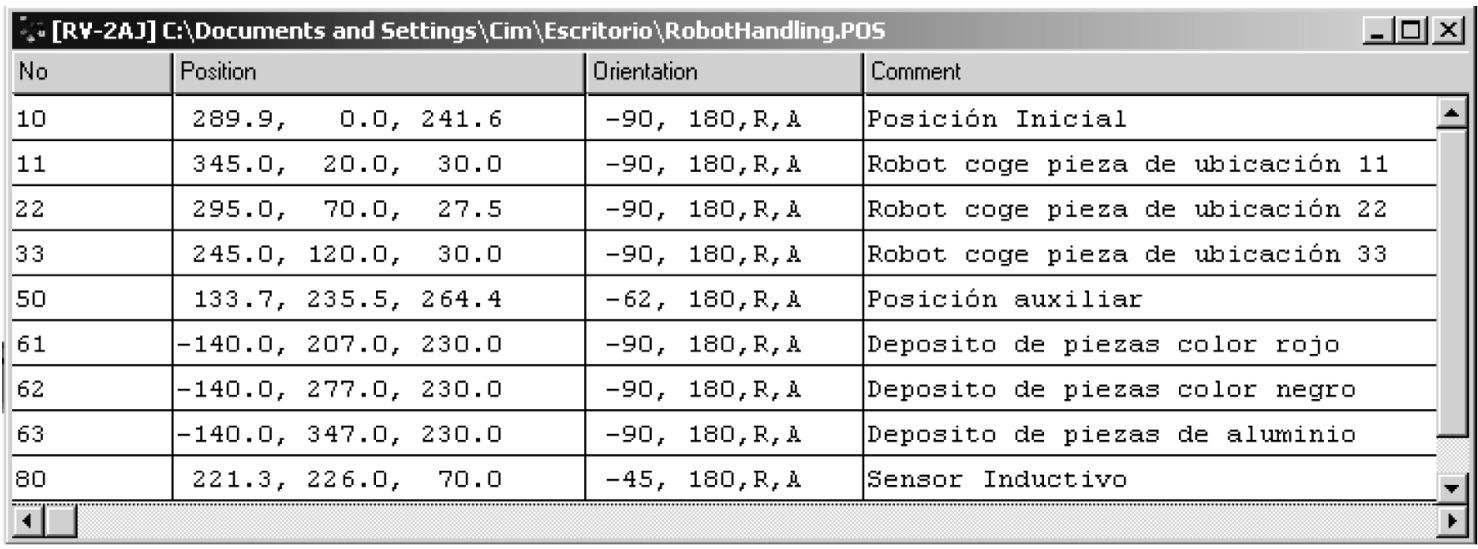

Fig. 5: Ventana de posiciones. 
Este software permite almacenar hasta un máximo de 999 posiciones. Para grabar posiciones el software dispone de un panel de operación llamado Jog Operation, el cual permite mover cada uno de los grados de libertad del robot y también variar su velocidad de desplazamiento. Los grados de libertad que pueden moverse se muestran en la Fig. 6 (Jog Operation): Waist (cintura), Shoulder (hombro), Elbow (codo), Pitch (elevación de muñeca) y Roll (giro de pinzas).

Si se desea mover solamente las articulaciones del robot se debe elegir la opción del modo Joint. En este caso se puede mover cualquiera de los cinco grados de libertad que posee el robot. El desplazamiento del robot en el modo Joint esta expresado en grados sexagesimales.

El modo $X Y Z$ se emplea cuando se requiere que el robot se mueva en el eje cartesiano. El modo $X Y Z$ se emplea para que el robot coja una pieza desde un plano horizontal ya que permite movimientos de arriba hacia abajo en el eje Z. El desplazamiento del robot en el modo $X Y Z$ está expresado en $\mathrm{mm}$.

El modo Tool se emplea cuando el robot va coger una pieza desde un plano inclinado ya que su coordenada $Z$ varía en función a la orientación que tiene la pinza del robot. Desde el panel de operaciones también se puede abrir y cerrar las pinzas del robot. El desplazamiento del robot en modo Tool también esta expresado en $\mathrm{mm}$.

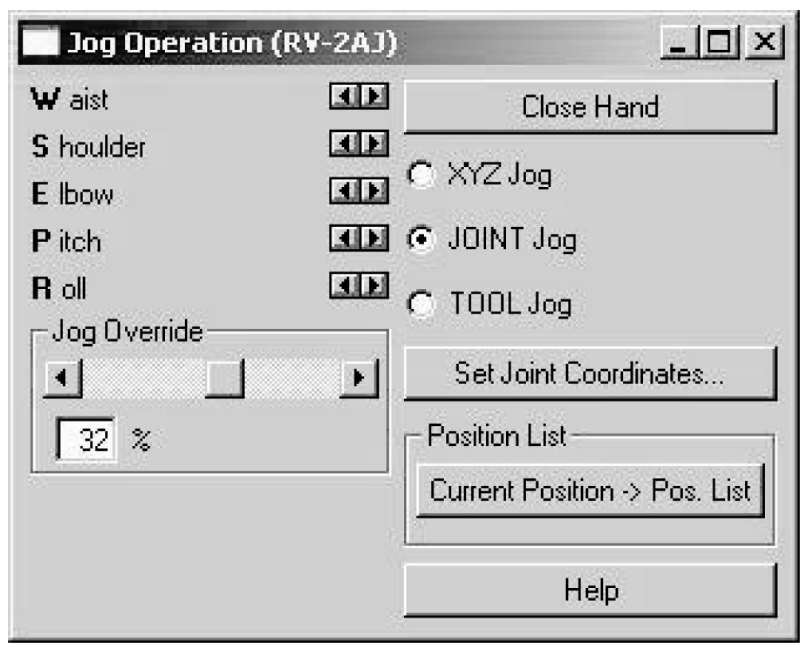

Fig. 6: Jog Operation.

\section{VENTANA DE PROGRAMACIÓN}

Desde la ventana de programación se puede crear la secuencia de acciones que va seguir el robot a lo largo de todo el proceso de producción. Las instruc- ciones o códigos que permiten al robot moverse contienen entre 2 y 3 caracteres. Las instrucciones comúnmente usadas son: MO (Move), MS (Move Straight), MTS (Move Tool Straight), DJ (Draw Joint), ED (End), GO (Grip Open), GC (Grip Close), IC (Increment counter), GS (Go Sub), MJ (Move Joint), SP (Speed), SC (Set Counter), TI (Timer), etc. Ver Fig. 7 (Ventana de programación).

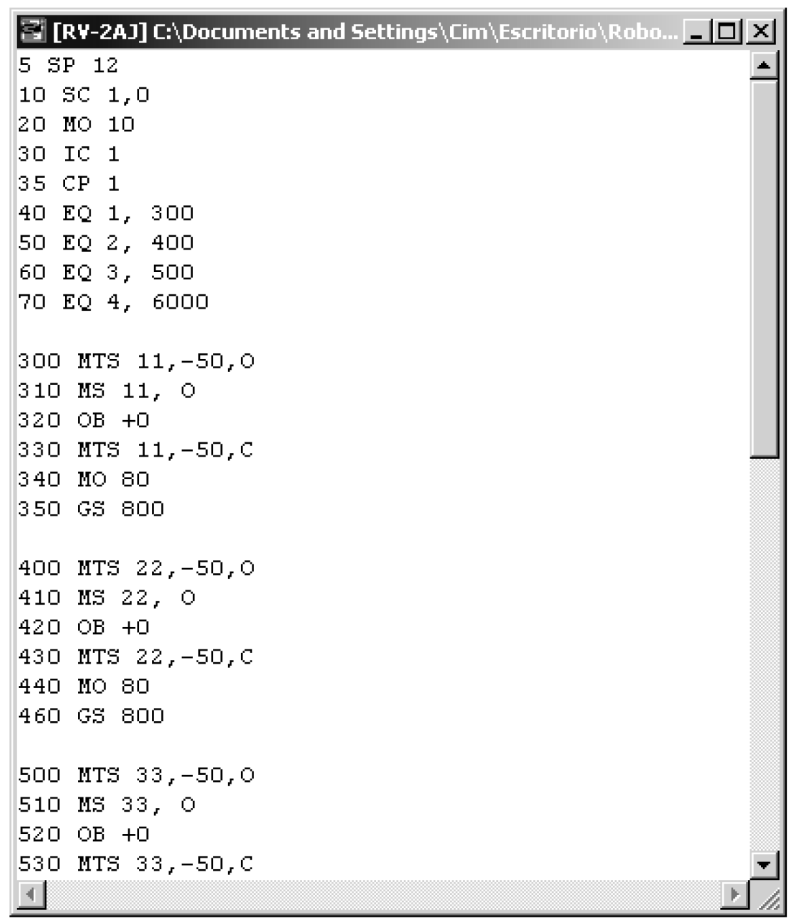

Fig. 7: Ventana de programación.

Existen también otras ventanas como la de mensajes, donde se muestran los errores y advertencias que puede tener el programa que se haya escrito en la ventana de programación. Posee además interfaces de entradas y salidas (inputs/outputs) que permiten conocer el estado en tiempo real de cada una de las señales que intervienen en el proceso. Una de las principales ventajas de este software es que constituye una poderosa herramienta para analizar nuevos escenarios de producción con nuevos productos, sin olvidar que también permite a los ingenieros de planta, simular y escoger la mejor alternativa de solución antes de probarlos en el sistema real.

\section{APLICACIÓN}

Se realizó la programación del robot para que a través de sensores pueda detectar el color y material de las piezas ubicadas en una bandeja, luego se las separó y ubicó en tres depósitos distintos. Ver Fig. 8 (Modelamiento de la aplicación). 
Para ello se dispone de una plataforma con lo siguiente:

- Un robot de cinco grados de libertad

- Una bandeja que contiene 3 piezas: 1 pieza de color rojo de plástico, 1 pieza de color negro mate de plástico y 1 pieza de aluminio.

- Un sensor óptico NC, para la detección de piezas de color negro, ubicado en la pinza del robot.

- Un sensor inductivo para detectar las piezas de aluminio.

- Cuatro depósitos de piezas, pero solo se utilizarán 3.

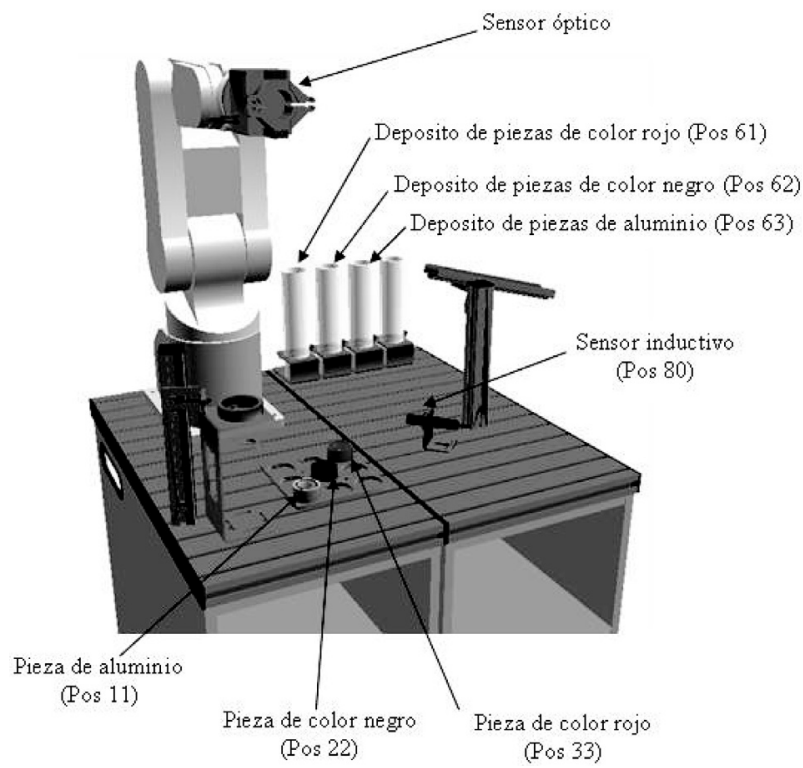

Fig. 8. Modelamiento de la aplicación.

Se grabaron 9 posiciones:

\begin{tabular}{|l|l|}
\hline Pos 10 & Posición Inicial \\
\hline Pos 11 & Robot coge pieza de ubicación 11 \\
\hline Pos 22 & Robot coge pieza de ubicación 22 \\
\hline Pos 33 & Robot coge pieza de ubicación 33 \\
\hline Pos 50 & Posición auxiliar \\
\hline Pos 61 & Depósito de piezas color rojo \\
\hline Pos 62 & Depósito de piezas color negro \\
\hline Pos 63 & Depósito de piezas de aluminio \\
\hline Pos 80 & Sensor Inductivo \\
\hline
\end{tabular}

A continuación se detalla la programación que se realizó empleando para ello contadores, comparadores, temporizadores y subrutinas:

\section{SP 30}

10 SC 1,0

$20 \mathrm{MO} 10$

30 IC 1

35 CP 1
40 EQ 1, 300

50 EQ 2, 400

60 EQ 3, 500

70 EQ 4, 6000

300 MTS 11,-50,0

310 MS 11,0

$320 \mathrm{OB}+0$

330 MTS $11,-50, C$

$340 \mathrm{MO} 80$

350 GS 800

400 MTS 22,-50,0

410 MS 22,0

420 OB +0

430 MTS 22,-50,C

440 MO 80

460 GS 800

500 MTS 33,-50,0

510 MS 33,0

$520 \mathrm{OB}+0$

530 MTS 33,-50,C

$540 \mathrm{MO} 80$

550 GS 800

800 TBD +3, 4000

810 TBD +2, 5000

820 GT 3000

3000 MO 50

3005 MTS 61,-10,C

3010 MS 61, C

3020 OB -0

3030 MTS 61,-10,0

3040 GT 20

4000 MO 50

4005 MTS 62,-10,C

4010 MS 62, C

4020 OB -0

4030 MTS 62, -10,0

4040 GT 20

5000 MO 50

5005 MTS 63,-10,C

5010 MS 63,C

5020 OB -0

5030 MTS 63,-10,0

5040 GT 20

6000 TI 10

6010 ED

\section{LIMITACIONES DEL SOFTWARE}

1. El software solo permite simular a través de un programa, la correcta secuencia que realiza el robot.

2. No se puede comunicar con el robot físicamente, para ello se requiere de otro software, con el que no solamente se logre simular una aplicación robotizada, sino que también se pueda 
descargar un programa y posiciones al controlador del robot para que pueda operar.

3. No existe una variedad de componentes en la librería del software.

4. Solamente se pueden programar, simular y modelar robots de marca FANUC, KUKA, Mitsubishi, Reis y Stäubli.

\section{CONCLUSIONES}

1. La simulación es bien reconocida entre los expertos profesionales como una herramienta que apoya a la mejora de la productividad de una empresa manufacturera o de servicios.

2. Cosimir Educational es un software bastante completo y potente que no solamente puede ser empleado por profesionales sino también por alumnos ya que es una excelente herramienta de modelamiento y simulación para procesos de fabricación automatizada.

3. Cosimir Educational no es el único software para simular procesos robotizados, existen varios otros disponibles en internet.

4. La ventana de modelamiento muestra todos los elementos que conforman el proceso robotizado.

5. La ventana de posiciones contiene coordenadas a través de las cuales el robot deberá desplazarse para que realice una tarea.
6. La ventana de programación emplea las posiciones para que el robot pueda desplazarse cumpliendo con los parámetros de programación como temporizadores, contadores, velocidad, aceleración, etc.

7. La programación de la aplicación se realizó correctamente, permitiendo que el robot decida en qué depósito colocar cada pieza.

\section{REFERENCIAS BIBLIOGRÁFICAS}

1. Cosimir Educational: http://www.festo-didactic. com/es-es/servicio-y-asistencia/printed-media/ manuales/manual-cosimir-educational-4.1.htm

2. Cosimir Educational. Getting Started. Cosimir Help.

3. La robótica y sus beneficios. Perfiles de Ingeniería. Universidad Ricardo Palma. 2003.

4. Industrial Robot Programming. Norberto Pires. 2007

5. Industrial robots: http://www.robotics.utexas.edu/ rrg/learn_more/low_ed/types/industrial.html 